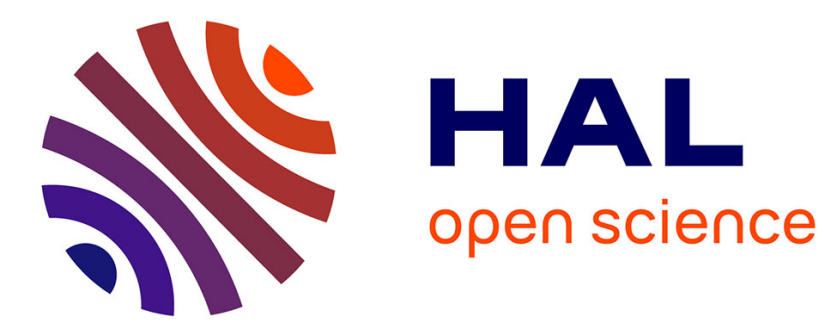

\title{
Density fitted, local Hartree-Fock treatment of NMR chemical shifts using London atomic orbitals
}

\author{
Stefan Loibl, Frederick R Manby, Martin Schütz
}

\section{To cite this version:}

Stefan Loibl, Frederick R Manby, Martin Schütz. Density fitted, local Hartree-Fock treatment of NMR chemical shifts using London atomic orbitals. Molecular Physics, 2010, 108 (03-04), pp.477-485. 10.1080/00268970903580133 . hal-00580691

\section{HAL Id: hal-00580691 https://hal.science/hal-00580691}

Submitted on 29 Mar 2011

HAL is a multi-disciplinary open access archive for the deposit and dissemination of scientific research documents, whether they are published or not. The documents may come from teaching and research institutions in France or abroad, or from public or private research centers.
L'archive ouverte pluridisciplinaire HAL, est destinée au dépôt et à la diffusion de documents scientifiques de niveau recherche, publiés ou non, émanant des établissements d'enseignement et de recherche français ou étrangers, des laboratoires publics ou privés. 


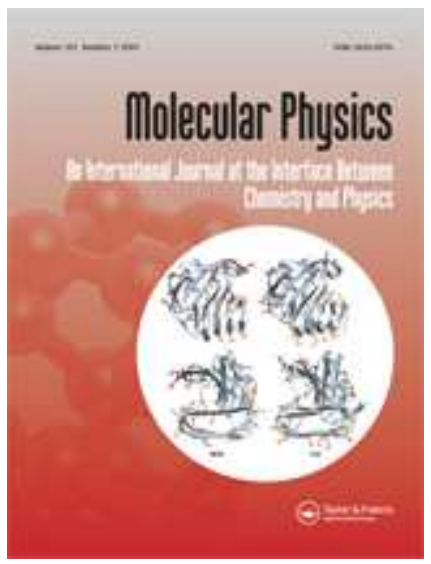

\section{Density fitted, local Hartree-Fock treatment of NMR chemical shifts using London atomic orbitals}

\begin{tabular}{|c|c|}
\hline Journal: & Molecular Physics \\
\hline Manuscript ID: & TMPH-2009-0279.R2 \\
\hline Manuscript Type: & Special Issue Paper - In honour of Prof Werner 60th birthday \\
\hline $\begin{array}{r}\text { Date Submitted by the } \\
\text { Author: }\end{array}$ & 17-Dec-2009 \\
\hline Complete List of Authors: & $\begin{array}{l}\text { Loibl, Stefan; University of Regensburg, Theoretical Chemistry } \\
\text { Manby, Frederick; University of Bristol, Centre for Computational } \\
\text { Chemistry, School of Chemistry } \\
\text { Schütz, Martin; University of Regensburg, Theoretical Chemistry }\end{array}$ \\
\hline Keywords: & GIAOs, NMR chemical shifts, density fitting, Hartree-Fock \\
\hline \multicolumn{2}{|c|}{$\begin{array}{l}\text { Note: The following files were submitted by the author for peer review, but cannot be converted } \\
\text { to PDF. You must view these files (e.g. movies) online. }\end{array}$} \\
\hline $\begin{array}{l}\text { giao_dfscf.tex } \\
\text { dirac.sty }\end{array}$ & \\
\hline
\end{tabular}

\section{ScholaroNE Manuscript Central}


During the last decade the calculation of NMR chemical shifts has become routinely applicable at the level of $a b$ initio electronic structure theory (for two more recent reviews see Refs. 1 and 2). One major obstacle to be overcome in such calculations is the gauge-origin problem arising from the incompleteness of the AO basis set. The Individual Gauge for Localized Orbitals (IGLOs) approach of Schindler and Kutzelnigg [3, 4] is one way to deal with that problem, however, nowadays, the preferred solution is the use of Gauge-Including Atomic Orbitals (GIAOs, or London atomic orbitals) $[5,6]$. A discussion about the pros and cons of IGLOs vs. GIAOs can be found in Refs. 7, 8, and, in the context of a DFT implementation, in Ref. 9. GIAOs depend explicitly on the external magnetic field and the gauge origin. In the expressions for the atomic integrals the latter dependence cancels, rendering the desired molecular properties independent of the gauge origin [10]. Efficient programs to calculate nuclear magnetic shielding tensors on the basis of GIAOs are presently available for Hartree-Fock (HF) and Density Functional Theory (DFT) [7, 11-13] and linear scaling methods have also been reported $[14,15]$. Yet, somewhat surprisingly, to our knowledge hardly any GIAO-HF or DFT implementation for the computation of NMR chemical shifts based on the Density Fitting (DF) [16-22] approximation for the Electron Repulsion Integrals (ERIs) has been reported so far. An exception may be the GIAO-DFT program by Schreckenbach and Ziegler (which is part of the Amsterdam density functional package $\mathrm{ADF}$ ), who mention in one sentence the use of auxiliary Slater type orbitals to fit the molecular density [12].

For a broad range of applications NMR chemical shifts computed at the HF or DFT level are sufficiently accurate. However, there are also many cases where a proper treatment of electron correlation, i.e., employing wavefunction based methods rather than DFT, is necessary (e.g. see sections 3.7, 3.8 in Ref. 2 and references therein). Correlated methods for calculating nuclear magnetic shielding constants with inclusion of dynamical electron correlation effects have been mainly reported for the GIAO approach. Gauss et al. presented Coupled Cluster (CC) GIAO implementations with CC models including up to triples and quadruples substitutions (see Refs. 23, 24 and references therein). Yet the application range of these methods is restricted to tiny molecules. Only the simplest electron correlation method, i.e., second-order Møller-Plesset perturbation theory (MP2) appears to be applicable to NMR chemical shift calculations of larger molecules. By virtue of integral-direct techniques [25] and full exploitation of non-Abelian point group symmetry [26] GIAO-MP2 chemical shift calculations of molecular systems comprising more than 600 basis functions could be performed [26]. However, due to the unfavorably high scaling of the computational cost with molecular size such calculations still are limited to molecules of relatively modest size or high symmetry.

From that perspective the development of correlated GIAO methods for chemical shift calculations based on local correlation approaches appears to be attractive. Efficient local correlation implementations ranging from MP2 to $\mathrm{CC}$ are available for the calculation of ground state energies [27-33] and gradients [34], as well as for properties of excited states [35-37]. A pilot implementation of GIAO local MP2 for chemical shifts has been presented by Gauss and Werner [38]. With this program, which simulates GIAO-LMP2 on top of a canonical GIAO-MP2 program, the

\footnotetext{
*martin.schuetz@chemie.uni-regensburg.de
} 
authors could asses the accuracy of GIAO-LMP2. They report that the effect of the local approximation on the resulting shifts are small (smaller than other errors inherent to $\mathrm{MP} 2$ itself), e.g., for ${ }^{13} \mathrm{C}$ smaller than 1 ppm, and conclude that "GIAO-LMP2 appears to be a powerful tool for chemical applications as soon as a computationally efficient code is available".

In this contribution we report a first step towards this goal. We present an efficient GIAO program for calculating nuclear magnetic shielding tensors based on density fitted Hartree-Fock (DF-HF). DF-HF is much more efficient than conventional HF when localized occupied orbitals are used in the construction of the exchange matrix. Furthermore, linear scaling can be achieved when restricting the fitting functions to local fit domains [39]. Also the efficient DFLMP2 analytic energy gradient (with respect to nuclear displacements) of the MOLPRO program package is based on the DF-HF energy functional (see section IIIB in Ref. 34). The GIAO-DF-HF method for chemical shifts employs Localized Molecular Orbitals (LMOs) and Projected Atomic Orbitals (PAOs) to span occupied and virtual space, respectively, in order to have sparse three-index ERIs and ERI derivatives in the Fock matrix constructions of the Coupled Perturbed Hartree-Fock step. LMOs are mutually orthogonal, while the PAOs are non-orthogonal and form an over-complete set. Ordinary Gaussians are employed as fitting functions for the orbital product densities that appear in the ERIs (since the use of GIAOs as fitting functions would trivially violate gauge origin independence of the ERIs). The error introduced by the fitting turns out to be negligible (see section III). The GIAO-DF-HF method for nuclear shieldings presented here is highly efficient, and, as for DF-HF, linear scaling could be achieved by introducing local fit domains. This work was originally considered as a necessary step towards an efficient GIAODF-LMP2 code. However, the GIAO-DF-HF program is interesting by itself because it makes it possible to calculate HF NMR chemical shifts efficiently within the MOLPRO program package.

\section{THEORY}

\section{A. The NMR chemical shielding tensor}

The NMR chemical shielding tensor is defined as the mixed second derivative of the electronic energy with respect to the components of the external magnetic field $\mathbf{B}$ and the magnetic moment $\mathbf{m}_{\mathbf{Z}}$ of nucleus $\mathbf{Z}$ :

$$
\sigma_{\beta \alpha}^{\mathrm{Z}} \equiv\left[\frac{d^{2} E_{\mathrm{HF}}}{d B_{\alpha} d m_{\mathrm{Z} \beta}}\right]_{\mathbf{B}, \mathbf{m}_{\mathbf{Z}}=\mathbf{0}} .
$$

For calculations in the context of the GIAO ansatz explicitly field-dependent basis functions $\omega_{\mu}\left(\mathbf{r}_{\mathrm{M}}, \mathbf{A}_{\mathrm{M}}\right)$ are used, i.e.,

$$
\omega_{\mu}\left(\mathbf{r}, \mathbf{A}_{\mathrm{M}}\right) \equiv \exp \left(-\frac{i}{c} \mathbf{A}_{\mathrm{M}} \cdot \mathbf{r}\right) \chi_{\mu}\left(\mathbf{r}_{\mathrm{M}}\right)
$$

where $\mathbf{A}_{\mathrm{M}}$ is the vector potential representing the magnetic field at nucleus $\mathrm{M}$

$$
\mathbf{A}_{\mathrm{M}}=\frac{1}{2} \mathbf{B} \times\left(\mathbf{R}_{\mathrm{M}}-\mathbf{R}_{\mathrm{O}}\right)=\frac{1}{2} \mathbf{B} \times \mathbf{R}_{\mathrm{MO}}
$$

and $\chi_{\mu}\left(\mathbf{r}_{\mathrm{M}}\right)$ the field-independent basis-functions. $\mathbf{R}_{\mathrm{M}}$ and $\mathbf{R}_{\mathrm{O}}$ represent the position vector of nucleus $\mathrm{M}$ and the gauge origin, $\mathbf{R}_{\mathrm{MO}}$ is their difference vector. Respectively, $\mathbf{r}$ denotes the position vector of an electron, and $\mathbf{r}_{\mathrm{M}}$ the vector pointing from nucleus $\mathrm{M}$ to this electron.

Greek letters $\mu, \nu, \ldots$ label atomic orbitals; $i, j, k, \ldots$ occupied localized molecular orbitals (LMOs); $a, b, c, \ldots$ projected atomic orbitals (PAOs); and $p$ general localized MOs. Orbital indices decorated with a bar, e.g. $\bar{i}, \bar{j}, \bar{k}, \ldots$ and $\bar{a}, \bar{b}, \bar{c}, \ldots$ denote canonical occupied and virtual orbitals, respectively.

The shielding tensor can be written more explicitly as

$$
\sigma_{\beta \alpha}^{\mathrm{Z}}=\left[\sum_{\mu \nu} D_{\mu \nu}^{0} \frac{\partial^{2} h_{\mu \nu}}{\partial B_{\alpha} \partial m_{\mathrm{Z} \beta}}+\sum_{\mu \nu} D_{\mu \nu}^{B_{\alpha}} \frac{\partial h_{\mu \nu}}{\partial m_{\mathrm{Z} \beta}}\right]_{\mathbf{B}=\mathbf{0}},
$$


with the unperturbed and (purely imaginary) perturbed density matrices

$$
\begin{aligned}
D_{\mu \nu}^{0} & \equiv 2 \sum_{i} L_{\mu i} L_{\nu i}, \\
D_{\mu \nu}^{B_{\alpha}} & \equiv 2 \sum_{i}\left[L_{\mu i}^{B_{\alpha}} L_{\nu i}+L_{\mu i} L_{\nu i}^{B_{\alpha} *}\right] .
\end{aligned}
$$

The derivatives of the LMO coefficients in eq. (6) can be expressed via the transformation matrix $\mathbf{U}^{B_{\alpha}}$, i.e.,

$$
L_{\mu i}^{B_{\alpha}}=\sum_{k} L_{\mu k} U_{k i}^{B_{\alpha}}+\sum_{c} P_{\mu c} U_{c i}^{B_{\alpha}}
$$

Here, the matrices $\mathbf{L}$ and $\mathbf{P}$ represent LMO coefficient matrix and the projection of the original AOs onto the virtual space, respectively (see e.g. eqs. $(2,3)$ in Ref. 40 ), and

$$
\mathbf{P}=\mathbf{1}-\mathbf{L L}^{\dagger} \mathbf{S}_{\mathrm{AO}}
$$

The one-electron derivative integrals appearing in eq. (4) can be written as

$$
\begin{aligned}
\frac{\partial^{2} h_{\mu \nu}}{\partial B_{\alpha} \partial m_{\mathrm{Z} \beta}}= & \frac{1}{2 c^{2}}\left[\left\langle\mu\left|\left(\mathbf{R}_{\mathrm{MN}} \times \mathbf{r}\right)_{\alpha} \frac{\left(\mathbf{r}_{\mathrm{Z}} \times \nabla\right)_{\beta}}{r_{\mathrm{Z}}^{3}}\right| \nu\right\rangle\right. \\
& \left.+\left\langle\mu\left|\frac{\left(\mathbf{r}_{\mathrm{N}} \cdot \mathbf{r}_{\mathrm{Z}}\right) \delta_{\alpha \beta}-\left(\mathbf{r}_{\mathrm{N}}\right)_{\alpha}\left(\mathbf{r}_{\mathrm{Z}}\right)_{\beta}}{r_{\mathrm{Z}}^{3}}\right| \nu\right\rangle\right], \\
\frac{\partial h_{\mu \nu}}{\partial m_{\mathrm{Z} \beta}}= & \frac{-i}{c}\left\langle\mu\left|\frac{\left(\mathbf{r}_{\mathrm{Z}} \times \nabla\right)_{\beta}}{r_{\mathrm{Z}}^{3}}\right| \nu\right\rangle .
\end{aligned}
$$

The explicit dependence of the integrals on the gauge origin $\mathbf{R}_{\mathrm{O}}$ cancels: both through the vector potential in the kinetic energy operator $\pi=-i \nabla+\mathbf{A}(r)$, as well as through the GIAOs cancels (for details, see e.g. Ref. 10). The derivative integrals in eqs. $(9,10)$ hence are independent of the choice of $\mathbf{R}_{\mathrm{O}}$. Furthermore, the derivative of the two-electron part does not occur in eq. (4) because it is independent of the perturbation $\mathbf{m}_{\mathrm{Z}}$. Since $\sigma_{\beta \alpha}^{\mathrm{Z}}$ is evaluated at zero field strength the GIAOs of the three one-electron integrals of eqs. $(9,10)$ reduce to ordinary Gaussian basis functions.

In order to construct the perturbed density matrix according to eq. (6) the general-occupied block of the transformation matrix $\mathbf{U}^{B_{\alpha}}$ has to be calculated. The requirement for preserving orthonormality of the orbitals spanning the occupied space, as well as strong orthogonality to the virtual space yields the condition

$$
U_{p i}^{B_{\alpha}}+U_{i p}^{B_{\alpha}^{*}}=-S_{p i}^{B_{\alpha}}
$$

with

$$
S_{\mu \nu}^{B_{\alpha}}=\frac{i}{2 c}\left\langle\mu\left|\left(\mathbf{R}_{\mathrm{MN}} \times \mathbf{r}\right)_{\alpha}\right| \nu\right\rangle
$$

Again, the $\mathbf{R}_{\mathrm{O}}$ dependence of the integral $S_{p q}^{B_{\alpha}}$ (here through the GIAOs only) trivially cancels.

Eq. (11) determines the occupied-occupied block of $\mathbf{U}^{B_{\alpha}}$ to be

$$
U_{i j}^{B_{\alpha}}=-\frac{1}{2} S_{i j}^{B_{\alpha}} .
$$

The virtual-occupied block of $\mathbf{U}^{B_{\alpha}}$ is determined by the requirement for preserving the Brillouin theorem and is obtained by solving the Coupled Perturbed Hartree-Fock (CPHF) equations

$$
\begin{aligned}
0 \stackrel{!}{=} R_{a i}= & \sum_{c} f_{a c} U_{c i}^{B_{\alpha}}-\sum_{k} f_{i k} U_{a k}^{B_{\alpha}} \\
& -\sum_{k} f_{i k} S_{a k}^{B_{\alpha}}+G\left(\mathbf{D}^{B_{\alpha}}\right)_{a i} \\
& +\left(\frac{\partial h_{a i}}{\partial B_{\alpha}}+\frac{\partial G\left(\mathbf{D}^{0}\right)_{a i}}{\partial B_{\alpha}}\right)
\end{aligned}
$$


involving the one- and two-electron derivative integrals

$$
\begin{aligned}
\frac{\partial h_{\mu \nu}}{\partial B_{\alpha}}= & \frac{i}{2 c}\left[\left\langle\mu\left|\left(\mathbf{R}_{\mathrm{MN}} \times \mathbf{r}\right)_{\alpha} h\right| \nu\right\rangle\right. \\
& \left.-\left\langle\mu\left|\left(\mathbf{r}_{\mathrm{N}} \times \nabla\right)_{\alpha}\right| \nu\right\rangle\right], \\
\frac{\partial\langle\mu \rho \mid \nu \sigma\rangle}{\partial B_{\alpha}}= & \frac{i}{2 c}\left[\left\langle\mu \rho\left|\frac{1}{r_{12}}\left(\mathbf{R}_{\mathrm{MN}} \times \mathbf{r}_{1}\right)_{\alpha}\right| \nu \sigma\right\rangle\right. \\
& \left.+\left\langle\mu \rho\left|\frac{1}{r_{12}}\left(\mathbf{R}_{\mathrm{RS}} \times \mathbf{r}_{2}\right)_{\alpha}\right| \nu \sigma\right\rangle\right] .
\end{aligned}
$$

Once more, the $\mathbf{R}_{\mathrm{O}}$ dependence cancels in these integrals, for the electron repulsion integral again trivially, since there $\mathbf{R}_{\mathrm{O}}$ occurs in all of the GIAOs and not in the operator.

To ensure fast convergence for the iterative solution of the CPHF equations the solution $\mathbf{U}^{B_{\alpha}}$ is not updated in the local LMO/PAO basis but in the canonical basis. The residual $\mathbf{R}$ is therefore transformed from the local to the canonical basis,

$$
R_{\bar{a} \bar{i}}=\sum_{a c i} Q_{\bar{a} c} V_{c a}^{\dagger} R_{a i} W_{i \bar{i}}^{\dagger},
$$

which is then used to generate the update for the new solution in each iteration step. The matrix $\mathbf{Q}$ is defined as

$$
\mathbf{Q}=\mathbf{C}^{\mathrm{v}} \mathbf{S}_{\mathrm{AO}}
$$

with $\mathbf{C}^{\mathrm{v}}$ and $\mathbf{S}_{\mathrm{AO}}$ representing the virtual block of the canonical MO coefficient matrix and the AO overlap matrix. $\mathbf{W}$ is the usual unitary transformation matrix specified by the localization criterion transforming canonical occupied orbitals $\phi_{i}^{\mathrm{CAN}}$ to LMOs $\phi_{i}^{\mathrm{LMO}}$. The matrix $\mathbf{V}$ is a pseudo-inverse of $\mathbf{S}_{\mathrm{PAO}}$ (see Ref. 41 ). $R_{\bar{a} \bar{i}}$ and $\mathbf{V}$ are sparse, however the second transformation step in eq. (17) involving $\mathbf{W}$ scales cubically, yet with a very low pre-factor. Therefore, the transformation of $\mathbf{R}$ to canonical basis does not present itself as a bottleneck, so far.

\section{B. Density fitting approximation for electron repulsion integrals}

For GIAO-DF-HF all ERIs are approximated by DF. Hence, for the calculation of the NMR chemical shielding tensor the expressions for the $G\left(\mathbf{D}^{B_{\alpha}}\right)$ and $\partial G\left(\mathbf{D}^{0}\right)_{\mu \nu} / \partial B_{\alpha}$ in the CPHF equations (14) have to be modified accordingly. The former is originally defined as

$$
\begin{aligned}
G\left(\mathbf{D}^{B_{\alpha}}\right)_{a i} & =\sum_{\rho \sigma} \mathbf{D}_{\rho \sigma}^{B_{\alpha}}\left((a i \mid \rho \sigma)-\frac{1}{2}(a \sigma \mid \rho i)\right) \\
& =-\frac{1}{2} \sum_{\rho \sigma} \mathbf{D}_{\rho \sigma}^{B_{\alpha}}(a \sigma \mid \rho i)
\end{aligned}
$$

where $(a i \mid \rho \sigma),(a \sigma \mid \rho i)$ represent the four-index ERIs (half-transformed to LMO/PAO basis), which are to be approximated by DF. For the last equality sign in the previous equation we have used the fact that $\mathbf{D}^{B_{\alpha}}$ is a purely imaginary hermitian matrix and as a consequence only the exchange part survives. Inserting eq. (6) yields

$$
G\left(\mathbf{D}^{B_{\alpha}}\right)_{a i}=-\sum_{k}[(a k \mid \tilde{k} i)-(a \tilde{k} \mid k i)],
$$

where $\tilde{k}$ denotes a perturbed LMO $\tilde{\chi}_{k}(\mathbf{r})$, transformed with the coefficient matrix $\mathbf{L}^{B_{\alpha}}$ defined in eq. (7).

The DF- approximation implies the expansion of the orbital product 'densities' $\chi_{k}(\mathbf{r}) \chi_{a}(\mathbf{r})$, $\tilde{\chi}_{k}(\mathbf{r}) \chi_{i}(\mathbf{r})$, etc. appearing in the ERIs in an auxiliary basis of fitting functions $\Xi_{P}(\mathbf{r})$. This leads to a factorization of the four-index ERIs in terms of three-index objects. From eq. (20) one so obtains

$$
G\left(\mathbf{D}^{B_{\alpha}}\right)_{a i}=-\sum_{k} \sum_{P}\left[c_{a k}^{P}(P \mid \tilde{k} i)-(a \tilde{k} \mid P) c_{k i}^{P}\right]
$$


with the three-index ERIs in LMO/PAO basis

$$
\begin{aligned}
(P \mid \tilde{k} i) & =\sum_{\mu \nu}(P \mid \mu \nu) L_{\mu k}^{B_{\alpha}} L_{\nu i}, \\
(a \tilde{k} \mid P) & =\sum_{\mu \nu} P_{\mu a} L_{\nu k}^{B_{\alpha}}(\mu \nu \mid P),
\end{aligned}
$$

and the fitting coefficients

$$
c_{p i}^{P}=\sum_{Q}\left[\mathbf{J}^{-1}\right]_{P Q}(Q \mid p i) .
$$

In eqs. (21-24) the indices $P, Q$ stand for auxiliary Fitting Functions (FFs), and $\mathbf{J}_{P Q}=(P \mid Q)$ is the Coulomb metric of these functions. Observe that the fitting coefficients $c_{p i}^{P}$ are independent of the CPHF iterations and thus just computed once. Furthermore, the three-index ERIs of eqs. (22-23) split into two parts according to eq. (7), with the first term being independent of the CPHF iterations, as well. The quantity $G\left(\mathbf{D}^{B_{\alpha}}\right)_{a i}$ of eqs. $(20,21)$ is, as any other term appearing in the CPHF equations (14), purely imaginary. The CPHF equations thus can be considered as real valued and solved for the imaginary part of the (purely imaginary) $\mathbf{U}^{B_{\alpha}}$.

An important note concerns the FFs. Using GIAOs as FFs would inevitably violate gauge invariance. Since for a given FF there is naturally no complex conjugate corresponding to the same electron the origin dependence (on $\mathbf{R}_{\mathrm{O}}$ ) would not cancel in the three-index ERIs. An alternative natural choice are ordinary Gaussian basis functions, implying that the GIAO orbital product densities are fitted at zero field, i.e., at $\mathbf{B}=\mathbf{0}$. In the present implementation no local restrictions to the fitting basis (fit domains [31, 33]) were yet introduced. However, since the orbital product 'densities' $\chi_{k}(\mathbf{r}) \chi_{a}(\mathbf{r}), \tilde{\chi}_{k}(\mathbf{r}) \chi_{i}(\mathbf{r})$, etc., are intrinsically local (provided that the solution of the CPHF equations $\mathbf{U}^{B_{\alpha}}$ entering eq. (7) is local), this could be accomplished. Substantial computational savings and eventually linear scaling of the computational cost can be expected for such a local fitting scheme. Since the individual local fitting basis sets then are different for the two orbital products in each ERI of eq. (20) the robust three-term formula has to be applied in the context of local fitting $[31,33]$ ), leading to four, rather than two contractions in eq. (21). The possibilities of local fitting in the context of GIAO-DF-HF will be explored in forthcoming work.

The $\partial G\left(\mathbf{D}^{0}\right)_{a i} / \partial B_{\alpha}$ part of the CPHF equations is also independent of the iteration and can be computed just once. Originally, it involves the contraction of the four-index derivative integrals defined in eq. (16) with the unperturbed density defined in eq. (5), i.e.,

$$
\frac{\partial G\left(\mathbf{D}^{0}\right)_{a i}}{\partial B_{\alpha}}=2 \sum_{k}\left[\frac{\partial(a i \mid k k)}{\partial B_{\alpha}}-\frac{1}{2} \frac{\partial(a k \mid k i)}{\partial B_{\alpha}}\right] .
$$

Invoking the DF-approximation yields

$$
\begin{aligned}
\frac{\partial G\left(\mathbf{D}^{0}\right)_{a i}}{\partial B_{\alpha}}= & \sum_{P} \widetilde{(a i \mid P)^{B}} c^{P} \\
& -\sum_{P k}\left(\widetilde{(a k \mid P)}^{B_{\alpha}} c_{k i}^{P}+c_{a k}^{P} \widetilde{(k i \mid P)}^{B_{\alpha}}\right),
\end{aligned}
$$

with

$$
c^{P}=\sum_{Q \rho \sigma} D_{\rho \sigma}^{0}\left[\mathbf{J}^{-1}\right]_{P Q}(\rho \sigma \mid Q),
$$

and

$$
\widetilde{(\mu \nu \mid P)}^{B_{\alpha}}=\frac{i}{2 c}\left(\mu \nu\left|\frac{1}{r_{12}}\left(\mathbf{R}_{\mathrm{MN}} \times \mathbf{r}_{\mathbf{1}}\right)_{\alpha}\right| P\right) .
$$

In the three-index derivative ERIs defined in eq. (28) the $\mathbf{R}_{\mathrm{O}}$ dependence of the GIAOs of the orbital product density cancels, while the FF does not carry any $\mathbf{R}_{\mathrm{O}}$ dependence by construction. Furthermore, due to the fact that these ERIs are needed at $\mathbf{B}=\mathbf{0}$ the GIAOs reduce to ordinary Gaussian basis functions. 


\section{TEST CALCULATIONS}

The new GIAO-DF-HF method for calculating nuclear magnetic shielding tensors is implemented in the MOLPRO package [42]. Most of the time-critical subroutines are parallelized, yet the scratch files containing the transformed three-index integrals and fitting coefficients reside on a common shared file system. In this section we present results from some test calculations, demonstrating accuracy and efficiency of the new program.

Tables I, II, and III compile the ${ }^{13} \mathrm{C},{ }^{17} \mathrm{O}$, and ${ }^{15} \mathrm{~N}$ chemical NMR shifts, calculated for a subset of the molecules used by Gauss in Ref. 43. The geometries of these molecules were optimized at the level of MP2 with the cc-pVTZ AO basis. GIAO-DF-HF calculations of the nuclear magnetic shielding tensors then were performed at these geometries, employing the cc-pVXZ AO basis, for $\mathrm{X}=\mathrm{D}, \mathrm{T}$, and Q, respectively. As fitting basis sets the JK-fitting basis sets of Weigend [44] related to cc-pVXZ, cc-pV(X+1)Z, and cc-pV(X+2)Z (if available) were used. Reference calculations were performed by using the conventional GIAO-HF implementation in TURBOMOLE [11]. From these results it is evident that the use of DF has virtually no effect on the resulting chemical shifts. Even without enlarging the cardinal number of the fitting basis relative to that of the AO basis the deviation relative to the reference value is in the far sub-ppm range, i.e., about $0.1 \mathrm{ppm}$ or less. The simple approach of employing ordinary Gaussians as fitting functions, or, in other words, to fit the orbital product densities in the ERIs at zero field (see discussion in section II B) hence appears to be the right way of applying DF in the context of property calculations involving GIAOs.

The efficiency of the GIAO-DF-HF program was explored by carrying out calculations on three somewhat more extended molecular systems, i.e. on coronene, on a tweezer host-guest complex 1@2 "clinching" the 1,4-dicyanobenzene guest molecule 2, and on a phenothiazine-isoalloxazine-pyrene triad (see Fig. 1). For the coronene molecule and the $1 @ 2$ tweezer experimental and theoretical results on NMR shifts are available in the literature ( $c f$. Refs. 45, 46 and references therein). For the calculations presented in this work the geometries of these molecules were optimized at the HF level using the SVP (coronene) and the 6-31G* (1@2) basis sets. Since the structure of the $1 @ 2$ tweezer so obtained deviates from that reported in Ref. 46, additional convential GIAO-HF calculations at the present structure were carried out for reference, rather than adopting the ${ }^{1} \mathrm{H}$ NMR shifts provided in Ref. 46. The triad has been used as a test molecule already in previous work [35, 37] and its structure has not been re-optimized for the present purpose.

Table IV contains the ${ }^{1} \mathrm{H}$ NMR shifts calculated with GIAO-DF-HF and a convential GIAO-HF in TURBOMOLE for coronene and 1@2 at their optimized geometries. The results of the GIAO-DF-HF calcaulation are essentially identical to the results of the GIAO-HF calculations. For the triad we just present the GIAO-DF-HF ${ }^{17} \mathrm{O}$, and ${ }^{15} \mathrm{~N}$ chemical shifts in Table V, a conventional reference calculation was not attempted for this case.

Tables IV and V also compile the CPU times (per processor) and the elapsed times, measured for the essential steps of the calculation, i.e., for the Fock build involving the ERI derivative integrals for the right-hand side of the CPHF equations according to eq. (26), and for an individual CPHF iteration. For comparison, the corresponding timings for an SCF iteration of the initial DF-HF calculation is also provided. The calculations were run on four cores, Intel(R) Xeon(R) CPU E5462 @ 2.80GHz, without exploiting any point group symmetry. For the calculation of the tweezer host-guest complex in the larger cc-pVTZ basis, solving the CPHF equations takes about 65 minutes elapsed to build up the right-hand side, plus about 30 minutes per iteration to converge the equations. An individual DF-HF SCF cycle, on the other hand, takes about 35 minutes. For the triad in the larger cc-pVTZ basis, a CPHF iteration costs about 140 minutes, which compares to 100 minutes for an SCF iteration. By comparing CPU and elapsed times in Table $\mathrm{V}$, it can be seen that I/O becomes a real bottleneck in our present parallel implementation. Yet there is certainly room for further optimization of the code in that respect.

\section{CONCLUSIONS}

In this contribution we present an efficient program for calculating nuclear magnetic shielding tensors at the level of density fitted (DF) Hartree-Fock for extended molecular systems. As basis functions Gauge-Including Atomic Orbitals (GIAOs, London atomic orbitals) are used. The orbital product densities appearing in the Electron Repulsion Integrals (ERIs) are expanded in a fitting basis consisting of ordinary Gaussians. GIAOs cannot be used here, since the dependence on the gauge origin would then not cancel in the ERIs. Using ordinary Gaussians as fitting functions corresponds to a fit of the orbital product densities at zero field strength of the external magnetic field. This simple approach works remarkably well: the dependence of the resulting chemical shifts on the size of the fitting basis set is virtually negligible and the agreement with results from conventional calculations without DF is typically within 
$0.1 \mathrm{ppm}$ or less. The efficiency of the new program is competitive, though some aspects could be further improved. This new program now provides the platform to develop a correlated GIAO method for nuclear magnetic shielding tensors based on DF Local MP2 [34], which is the primary target of this development.

\section{Acknowledgments}

S.L. gratefully acknowledges a scholarhip from the German National Academic Foundation.

[1] T. Helgaker, M. Jaszunski and K. Ruud, Chem. Rev. 99, 293 (1999).

[2] J. Gauss, Molecular Properties, in Modern Methods and Algorithms of Quantum Chemistry, edited by J. Grotendorst, no. 3 in NIC Series (, , 2000), p. 541.

[3] M. Schindler and W. Kutzelnigg, J .Chem .Phys. 76 (4), 1919 (1982).

[4] U. Meier, C. van Wüllen and M. Schindler, J. Comput. Chem. 13 (5), 551 (1992).

[5] F. London, J. Phys. Radium 8 (10), 397 (1937).

[6] R. Ditchfield, Mol. Phys. 27 (4), 789 (1974).

[7] K. Wolinski, J. Hinton and P. Pulay, J. Am. Chem. Soc. 112 (23), 8251 (1990).

[8] K. Ruud, T. Helgaker, K. BAK, P.Jørgensen and H. Jensen, J. Chem. Phys. 99 (5), 3847 (1993).

[9] C. van Wüllen, J. Chem. Phys. 102 (7), 2806 (1995).

[10] T. Helgaker and P. Jørgensen, J. Chem. Phys. 95 (4), 2595 (1991).

[11] M. Häser, R. Ahlrichs, H. Baron, P. Weis and H. Horn, Theor. Chim. Acta 83, 455 (1992).

[12] G. Schreckenbach and T. Ziegler, J. Phys. Chem. 99 (2), 606 (1995).

[13] G. Rauhut, S. Puyear, K. Wolinski and P. Pulay 1996.

[14] J. Kussmann and C. Ochsenfeld, J. Chem. Phys. 127 (5), 054103 (2007).

[15] C. Ochsenfeld, J. Kussmann and F. Koziol, Angew. Chem. Int. Ed. 43, 4485 (2004).

[16] E.J. Baerends, D.E. Ellis and P. Ros, Chem. Phys. 2, 41 (1973).

[17] J.L. Whitten, J. Chem. Phys. 58, 4496 (1973).

[18] B.I. Dunlap, J.W.D. Connolly and J.R. Sabin, J. Chem. Phys. 71, 3396 (1979).

[19] M. Häser and J. Almlöf, J. Chem. Phys. 96, 489 (1992).

[20] M. Feyereisen, G. Fitzgerald and A. Komornicki, Chem. Phys. Letters 208, 359 (1993).

[21] F. Weigend and M. Häser, Theor. Chim. Acta 97, 331 (1997).

[22] F. Weigend, M. Häser, H. Patzelt and R. Ahlrichs, Chem. Phys. Lett. 294, 143 (1998).

[23] J. Gauss, J. Chem. Phys. 116 (12), 4773 (2002).

[24] M. Kallay and J. Gauss, J. Chem. Phys. 120 (15), 6841 (2004).

[25] M. Kollwitz and J. Gauss, Chem. Phys. Letters 260, 639 (1996).

[26] M. Kollwitz, M. Häser and J. Gauss, J. Chem. Phys. 108 (20), 8295 (1998).

[27] M. Schütz, G. Hetzer and H.J. Werner, J. Chem. Phys. 111, 5691 (1999).

[28] M. Schütz and H.J. Werner, J. Chem. Phys. 114, 661 (2001).

[29] M. Schütz, J. Chem. Phys. 113, 9986 (2000).

[30] M. Schütz, J. Chem. Phys. 116, 8772 (2002).

[31] M. Schütz and F.R. Manby, Phys. Chem. Chem. Phys. 5, 3349 (2003).

[32] T. Adler, H.J. Werner and F.R. Manby, J. Chem. Phys. 130, 054106 (2009).

[33] H.J. Werner, F.R. Manby and P.J. Knowles, J. Chem. Phys. 118, 8149 (2003).

[34] M. Schütz, H.J. Werner, R. Lindh and F.R. Manby, J. Chem. Phys. 121, 737 (2004).

[35] D. Kats, T. Korona and M. Schütz, J. Chem. Phys. 125, 104106 (2006).

[36] D. Kats, T. Korona and M. Schütz, J. Chem. Phys. 127, 064107 (2007).

[37] D. Kats and M. Schütz, J. Chem. Phys. 131, 124117 (2009).

[38] J. Gauss and H.J. Werner, Phys. Chem. Chem. Phys. 2 (10), 2083 (2000).

[39] R. Polly, H.J. Werner, F.R. Manby and P.J. Knowles, Mol. Phys. 102, 2311 (2004).

[40] M. Schütz, G. Hetzer and H.J. Werner, J. Chem. Phys. 111, 5691 (1999).

[41] D. Kats, D. Usvyat and M. Schütz, Phys. Chem. Chem. Phys. 10, 3430 (2008).

[42] H.J. Werner, P.J. Knowles, R. Lindh, F.R. Manby, M. Schütz et al., MOLPRO, version 2009.2, a package of ab initio programs Cardiff, UK, 2009, see http://www.molpro.net.

[43] J. Gauss, J. Chem. Phys. 99 (5), 3629 (1993).

[44] F. Weigend, A fully direct RI-HF algorithm: optimized auxiliary basis sets, demonstration of accuracy and efficiencyPhys. Chem. Chem. Phys. 4, 4285 (2002).

[45] C. Ochsenfeld, Phys. Chem. Chem. Phys. 2 (10), 2153 (2000). 
[46] S. Brown, T. Schaller, U. Seelbach, F. Koziol, C. Ochsenfeld, F. Klärner and H. Spiess, Angew. Chem. Int. Edit. 40 (4), 717 (2001).

FIG. 1: Example molecules, (i) coronene, (ii) tweezer host-guest complex $1 @ 2$ with 1,4-dicyanobenzene as guest molecule 2 and (iii) phenothiazine-isoalloxazine-pyrene triad.

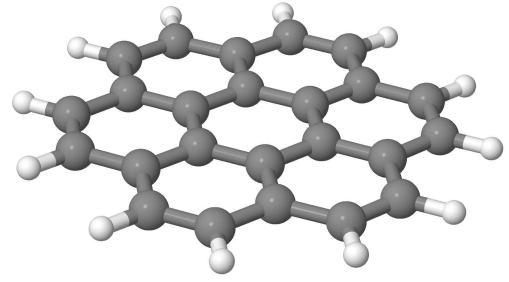

(i) coronene, 36 atoms, 156 electrons 396/1956 basis/fit functions (cc-pVDZ) $888 / 2256$ basis/fit functions (cc-pVTZ)

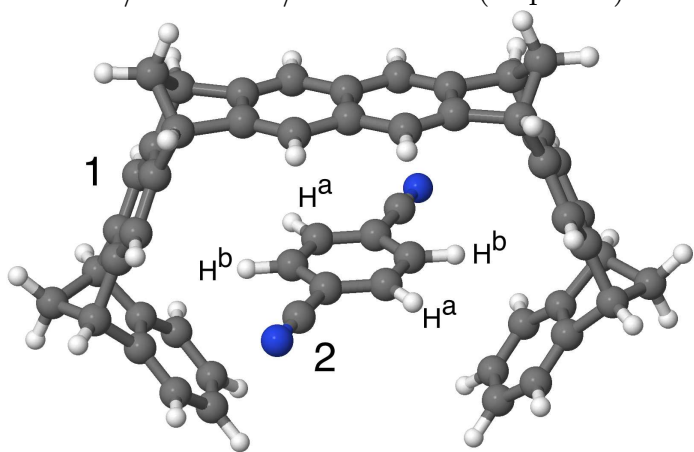

(ii) tweezer host-guest complex $1 @ 2$, with 2=1,4-dicyanobenzene, 92 atoms, 374 electrons

964/4748 basis/fit functions (cc-pVDZ) 2184/5504 basis/fit functions (cc-pVTZ)

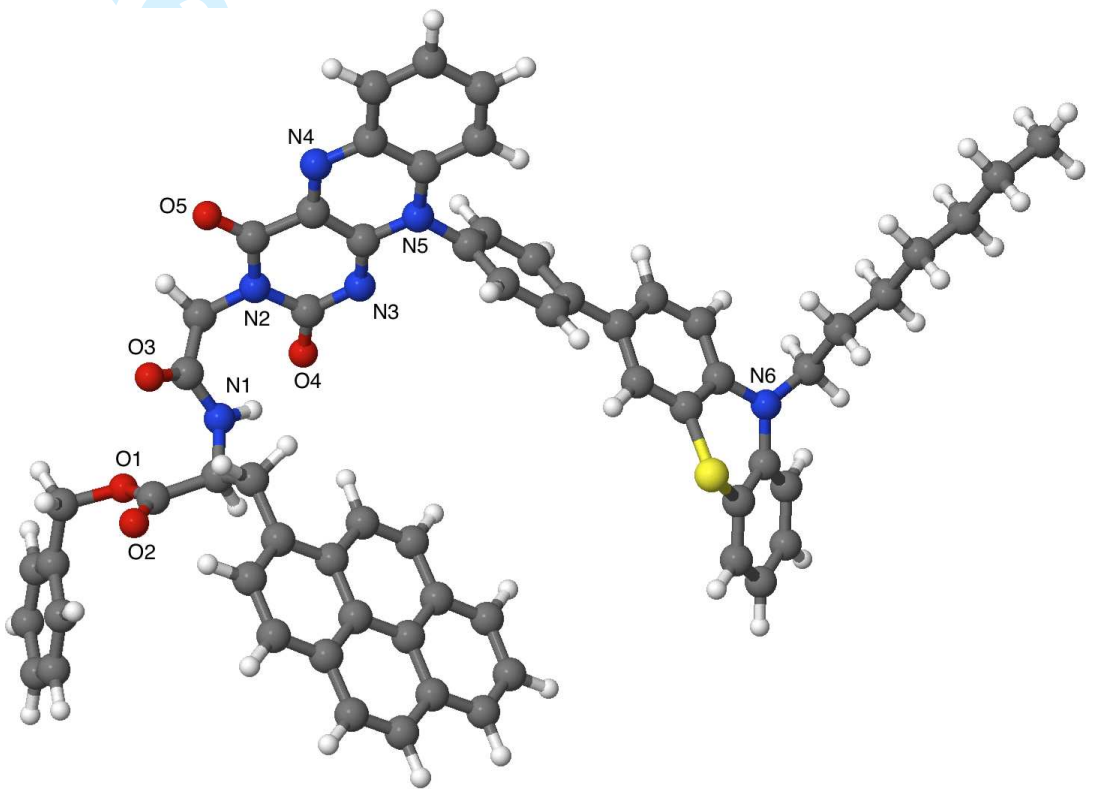

(iii) phenothiazine-isoalloxazine-pyrene triad, 127 atoms, 528 electrons $1314 / 6488$ basis/fit functions (cc-pVDZ) 2982/7527 basis/fit functions (cc-pVTZ) 


\section{Page 9 of 12}

Molecular Physics

1

3

4

5

6

7

8

9

10

11

12

13

14

15

16

17

18

19

20

21

22

23

24

25

26

27

28

29

30

31

32

33

34

35

36

37

38

39

40

41

42

43

44

45

46

47

48

49

50

51

52

53

54

55

56

57

58

59

60

TABLE I: ${ }^{13} \mathrm{C}$ chemical shifts $(\delta$, ppm) obtained with the new GIAO-DF-HF program, and compared to conventional GIAO-HF reference values. All shifts relative to $\mathrm{CH}_{4}{ }^{a}$ as in Ref. 43 .

\begin{tabular}{|c|c|c|c|c|c|c|c|c|c|c|c|}
\hline \multirow{2}{*}{$\begin{array}{l}\text { Basis } \\
\text { Fit basis }\end{array}$} & \multicolumn{4}{|c|}{ cc-pVDZ } & \multicolumn{4}{|c|}{ cc-pVTZ } & \multicolumn{3}{|c|}{ cc-pVQZ } \\
\hline & VDZ & VTZ & VQZ & ref. $^{b}$ & VTZ & VQZ & V5Z & ref. $^{b}$ & VQZ & V5Z & ref. $^{b}$ \\
\hline $\mathrm{C}_{6} \mathrm{H}_{6}$ & 134.14 & 134.12 & 134.13 & 134.14 & 138.48 & 138.49 & 138.49 & 138.50 & 140.45 & 140.45 & 140.45 \\
\hline $\mathrm{CF}_{4}$ & 109.92 & 109.88 & 109.88 & 109.89 & 115.42 & 115.42 & 115.41 & 115.41 & 116.11 & 116.11 & 116.11 \\
\hline $\mathrm{CH}_{3} \mathrm{~F}$ & 64.51 & 64.49 & 64.49 & 64.50 & 69.22 & 69.22 & 69.22 & 69.22 & 69.90 & 69.90 & 69.90 \\
\hline $\mathrm{C}_{2} \mathrm{H}_{2}$ & 74.51 & 74.50 & 74.50 & 74.51 & 79.26 & 79.26 & 79.26 & 79.26 & 80.18 & 80.18 & 80.18 \\
\hline $\mathrm{C}_{2} \mathrm{H}_{4}$ & 128.37 & 128.36 & 128.37 & 128.38 & 133.09 & 133.09 & 133.09 & 133.10 & 135.25 & 135.25 & 135.26 \\
\hline $\mathrm{CH}_{2} \mathrm{O}$ & 193.30 & 193.27 & 193.27 & 193.27 & 200.50 & 200.48 & 200.48 & 200.48 & 203.52 & 203.51 & 203.52 \\
\hline$\left(\mathrm{CH}_{3}\right)_{2} \underline{\mathrm{CO}}$ & 211.25 & 211.19 & 211.19 & 211.20 & 214.74 & 214.72 & 214.72 & 214.73 & 219.02 & 219.01 & 219.02 \\
\hline$\left(\underline{\mathrm{CH}}_{3}\right)_{2} \mathrm{CO}$ & 29.70 & 29.69 & 29.70 & 29.71 & 31.21 & 31.21 & 31.21 & 31.22 & 31.90 & 31.89 & 31.91 \\
\hline $\mathrm{CH}_{3} \mathrm{CH}_{3}$ & 10.95 & 10.93 & 10.93 & 10.94 & 11.38 & 11.38 & 11.38 & 11.38 & 11.91 & 11.91 & 11.91 \\
\hline $\mathrm{CH}_{3} \underline{\mathrm{CN}}$ & 125.03 & 124.99 & 125.00 & 125.01 & 131.96 & 131.95 & 131.95 & 131.96 & 134.61 & 134.61 & 134.62 \\
\hline$\underline{\mathrm{CH}_{3}} \mathrm{CN}$ & 4.18 & 4.17 & 4.17 & 4.18 & 4.10 & 4.09 & 4.09 & 4.11 & 4.49 & 4.49 & 4.50 \\
\hline$\underline{\mathrm{C}} \mathrm{H}_{3} \mathrm{CHO}$ & 30.95 & 30.94 & 30.95 & 30.96 & 32.49 & 32.49 & 32.49 & 32.49 & 33.41 & 33.41 & 33.42 \\
\hline $\mathrm{CH}_{3} \underline{\mathrm{C}} \mathrm{HO}$ & 201.07 & 201.03 & 201.03 & 201.03 & 206.89 & 206.87 & 206.87 & 206.88 & 210.48 & 210.47 & 210.49 \\
\hline $\mathrm{CH}_{3} \mathrm{NH}_{2}$ & 29.77 & 29.76 & 29.76 & 29.77 & 31.27 & 31.27 & 31.27 & 31.28 & 31.99 & 31.99 & 32.00 \\
\hline $\mathrm{CH}_{3} \mathrm{OH}$ & 47.46 & 47.44 & 47.44 & 47.45 & 50.69 & 50.69 & 50.69 & 50.70 & 51.68 & 51.68 & 51.69 \\
\hline $\mathrm{CO}_{2}$ & 136.35 & 136.30 & 136.30 & 136.31 & 145.40 & 145.38 & 145.38 & 145.38 & 146.59 & 146.59 & 146.59 \\
\hline $\mathrm{CO}$ & 214.29 & 214.25 & 214.25 & 214.28 & 222.24 & 222.21 & 222.20 & 222.23 & 225.16 & 225.15 & 225.18 \\
\hline
\end{tabular}

a) Absolute chemical shifts for $\mathrm{CH}_{4}: 205.36$ (cc-pVDZ/cc-pVDZ), 205.37 (cc-pVDZ/cc-pVTZ), 205.38 (cc-pVDZ/cc-pVQZ), 205.38 (ref., cc-pVDZ), 196.84 (cc-pVTZ/cc-pVTZ), 196.84 (cc-pVTZ/cc-pVQZ), 196.84 (cc-pVTZ/cc-pV5Z), 196.84 (ref., ccpVTZ), 195.66 (cc-pVQZ/cc-pVQZ), 195.66 (cc-pVQZ/cc-pV5Z), 195.66 (ref., cc-pVQZ). The basis sets are specified as (AO basis / corresponding JKfit basis).

b) GIAO HF values calculated with TURBOMOLE. 
TABLE II: ${ }^{17} \mathrm{O}$ chemical shifts $(\delta$, ppm) obtained with the new GIAO-DF-HF program, and compared to conventional GIAO-HF reference values. All shifts relative to $\mathrm{H}_{2} \mathrm{O}^{a}$ as in Ref. 43 .

\begin{tabular}{|c|c|c|c|c|c|c|c|c|c|c|c|}
\hline \multirow{2}{*}{$\begin{array}{l}\text { Basis } \\
\text { Fit basis }\end{array}$} & \multicolumn{4}{|c|}{ cc-pVDZ } & \multicolumn{4}{|c|}{ cc-pVTZ } & \multicolumn{3}{|c|}{ cc-pVQZ } \\
\hline & VDZ & VTZ & VQZ & ref. $^{b}$ & VTZ & VQZ & V5Z & ref. $^{b}$ & VQZ & V5Z & ref. $^{b}$ \\
\hline $\mathrm{CH}_{3} \mathrm{OH}$ & -5.37 & -5.41 & -5.42 & -5.42 & -8.67 & -8.68 & -8.68 & -8.67 & -11.07 & -11.07 & -11.06 \\
\hline $\mathrm{H}_{2} \mathrm{O}_{2}$ & 189.58 & 189.57 & 189.55 & 189.58 & 188.75 & 188.74 & 188.74 & 188.75 & 187.13 & 187.13 & 187.14 \\
\hline $\mathrm{CO}$ & 417.66 & 417.59 & 417.57 & 417.56 & 429.26 & 429.21 & 429.21 & 429.20 & 426.69 & 426.68 & 426.67 \\
\hline $\mathrm{CO}_{2}$ & 120.44 & 120.36 & 120.34 & 120.35 & 120.84 & 120.80 & 120.81 & 120.82 & 116.10 & 116.15 & 116.16 \\
\hline $\mathrm{CH}_{2} \mathrm{O}$ & 784.19 & 784.04 & 784.00 & 784.00 & 785.14 & 785.16 & 785.13 & 785.13 & 781.00 & 781.14 & 781.10 \\
\hline $\mathrm{CH}_{3} \mathrm{CHO}$ & 700.69 & 700.58 & 700.58 & 700.57 & 700.96 & 700.93 & 700.93 & 700.92 & 697.56 & 697.50 & 697.44 \\
\hline$\left(\mathrm{CH}_{3}\right)_{2} \mathrm{CO}$ & 669.37 & 669.30 & 669.31 & 669.32 & 669.73 & 669.69 & 669.70 & 669.69 & 667.06 & 666.99 & 666.95 \\
\hline
\end{tabular}

a) Absolute chemical shifts for $\mathrm{H}_{2} \mathrm{O}: 347.16$ (cc-pVDZ/cc-pVDZ), 347.18 (cc-pVDZ/cc-pVTZ), 347.18 (cc-pVDZ/cc-pVQZ), 347.19 (ref., cc-pVDZ), 334.90 (cc-pVTZ/cc-pVTZ), 334.89 (cc-pVTZ/cc-pVQZ), 334.90 (cc-pVTZ/cc-pV5Z), 334.91 (ref., ccpVTZ), 330.74 (cc-pVQZ/cc-pVQZ), 330.74 (cc-pVQZ/cc-pV5Z), 330.75 (ref., cc-pVQZ). The basis sets are specified as (AO basis / corresponding JKfit basis).

b) GIAO HF values calculated with TURBOMOLE.

TABLE III: ${ }^{15} \mathrm{~N}$ chemical shifts $(\delta$, ppm) obtained with the new GIAO-DF-HF program, and compared to conventional GIAOHF reference values. All shifts relative to $\mathrm{NH}_{3}{ }^{a}$ as in Ref. 43.

\begin{tabular}{|c|c|c|c|c|c|c|c|c|c|c|c|}
\hline \multirow{2}{*}{$\begin{array}{l}\text { Basis } \\
\text { Fit basis }\end{array}$} & \multicolumn{4}{|c|}{ cc-pVDZ } & \multicolumn{4}{|c|}{ cc-pVTZ } & \multicolumn{3}{|c|}{ cc-pVQZ } \\
\hline & VDZ & VTZ & VQZ & ref. $^{b}$ & VTZ & VQZ & V5Z & ref. $^{b}$ & VQZ & $\mathrm{V} 5 \mathrm{Z}$ & ref. $^{b}$ \\
\hline $\mathrm{HCN}$ & 309.85 & 309.80 & 309.77 & 309.77 & 322.86 & 322.85 & 322.85 & 322.85 & 322.57 & 322.56 & 322.56 \\
\hline $\mathrm{CH}_{3} \mathrm{NH}_{2}$ & 14.29 & 14.27 & 14.26 & 14.26 & 14.82 & 14.81 & 14.82 & 14.83 & 13.47 & 13.47 & 13.47 \\
\hline $\mathrm{CH}_{3} \mathrm{CN}$ & 302.10 & 302.06 & 302.05 & 302.00 & 313.92 & 313.90 & 313.90 & 313.85 & 313.38 & 313.38 & 313.34 \\
\hline $\mathrm{N}_{2}$ & 383.64 & 383.54 & 383.52 & 383.51 & 393.16 & 393.12 & 393.13 & 393.12 & 393.78 & 393.78 & 393.78 \\
\hline
\end{tabular}

a) Absolute chemical shifts for $\mathrm{NH}_{3}: 278.27$ (cc-pVDZ/cc-pVDZ), 278.28 (cc-pVDZ/cc-pVTZ), 278.28 (cc-pVDZ/cc-pVQZ), 278.28 (ref., cc-pVDZ), 269.18 (cc-pVTZ/cc-pVTZ), 269.17 (cc-pVTZ/cc-pVQZ), 269.17 (cc-pVTZ/cc-pV5Z), 269.18 (ref., ccpVTZ), 265.51 (cc-pVQZ/cc-pVQZ), 265.51 (cc-pVQZ/cc-pV5Z), 265.51 (ref., cc-pVQZ). The basis sets are specified as (AO basis / corresponding JKfit basis).

b) GIAO HF values calculated with TURBOMOLE. 
Molecular Physics

1

TABLE IV: GIAO-DF-HF ${ }^{1} \mathrm{H}$ chemical shifts $\delta$ (in ppm) calculated for the tweezer host-guest complex $1 @ 2$ and the coronene molecule, respectively, along with the corresponding conventional reference values. The shifts are given relative to TMS reference $a b$. In addition, the CPU times (per processor) and the elapsed times (in minutes) measured for the individual key steps of the GIAO-DF-HF calculation, i.e., the timings for the setup of the rhs of the CPHF equations, and the averaged CPHF iteration times are given. For comparison, the averaged SCF iteration times of the initial DF-HF calculation are also provided.

\begin{tabular}{|c|c|c|c|c|}
\hline Basis & \multicolumn{2}{|l|}{ cc-pVDZ } & \multicolumn{2}{|c|}{ cc-pVTZ } \\
\hline \multicolumn{5}{|c|}{ Tweezer host-guest complex 1@2 $2^{a}$} \\
\hline & GIAO-DF-HF & ref. $^{c}$ & GIAO-DF-HF & ref. $^{c}$ \\
\hline $\mathrm{H}^{a}$ (guest) & 5.8 & 5.8 & 5.8 & 5.8 \\
\hline $\mathrm{H}^{b}$ (guest) & 3.7 & 3.7 & 3.5 & 3.5 \\
\hline H $2,3,14,15$ & 6.7 & 6.7 & 6.6 & 6.6 \\
\hline $\mathrm{H}_{\text {arom }}($ host $)$ & $7.2-7.8$ & $7.2-7.8$ & $7.3-7.8$ & $7.3-7.8$ \\
\hline $\mathrm{H}_{\text {bridgehead }}$ & $3.7-4.0$ & $3.7-4.0$ & $3.9-4.1$ & $3.9-4.1$ \\
\hline H 25,28 & 2.2 & 2.2 & 2.2 & 2.2 \\
\hline H 26,27 & $1.9-2.1$ & $1.9-2.1$ & $2.0-2.1$ & $2.0-2.1$ \\
\hline \multicolumn{5}{|l|}{ Building eq. (26) } \\
\hline CPU (elapsed) time / min & \multicolumn{2}{|c|}{$13.4(13.4)$} & \multicolumn{2}{|c|}{$65.0(65.2)$} \\
\hline \multicolumn{5}{|l|}{ CPHF iteration step } \\
\hline CPU (elapsed) time / min & \multicolumn{2}{|c|}{$10.8(11.0)$} & \multicolumn{2}{|c|}{$28.7(29.0)$} \\
\hline \multicolumn{5}{|l|}{ DF-HF iteration step } \\
\hline CPU (elapsed) time / min & \multicolumn{2}{|l|}{$9.4(9.5)$} & \multicolumn{2}{|c|}{$33.2(33.4)$} \\
\hline \multicolumn{5}{|l|}{ Coronene $^{b}$} \\
\hline & GIAO-DF-HF & ref. $^{c}$ & GIAO-DF-HF & ref. $^{c}$ \\
\hline $\mathrm{H}$ & 9.0 & 9.0 & 9.2 & 9.2 \\
\hline \multicolumn{5}{|l|}{ Building eq. (26) } \\
\hline $\begin{array}{l}\text { CPU (elapsed) time / min } \\
\text { CPHF iteration step }\end{array}$ & \multicolumn{2}{|l|}{$0.9(0.9)$} & \multicolumn{2}{|c|}{$3.3(3.3)$} \\
\hline CPU (elapsed) time / min & \multicolumn{2}{|l|}{$0.6(0.6)$} & \multicolumn{2}{|c|}{$2.1(2.1)$} \\
\hline \multicolumn{5}{|l|}{ DF-HF iteration step } \\
\hline CPU (elapsed) time / min & \multicolumn{2}{|l|}{$0.4(0.4)$} & \multicolumn{2}{|c|}{$1.1(1.1)$} \\
\hline
\end{tabular}

a) TMS optimized at HF $/ 6-31 \mathrm{G}^{*}$ level, absolute chemical shielding $32.2 \mathrm{ppm}$ (cc-pVDZ), $32.1 \mathrm{ppm}$ (cc-pVTZ).

b) TMS optimized at HF/SVP level, absolute chemical shieldings 32.0 ppm (cc-pVDZ), 31.9 (cc-pVTZ).

c) GIAO HF values calculated with TURBOMOLE. 
TABLE V: GIAO-DF-HF relative chemical shifts (in ppm) for the heteroatoms of the phenothiazine-isoalloxazine-pyrene triad. ${ }^{15} \mathrm{~N}$ chemical shifts are relative to $\mathrm{NH}_{3}{ }^{a},{ }^{17} \mathrm{O}$ chemical shifts are relative to $\mathrm{H}_{2} \mathrm{O}{ }^{b}$. In addition, the CPU times (per processor) and the elapsed times (in minutes) measured for the individual key steps of the GIAO-DF-HF calculation, i.e., the timings for the setup of the rhs of the CPHF equations, and the averaged CPHF iteration times are given. For comparison, the averaged SCF iteration times of the initial DF-HF calculation are also provided.

\begin{tabular}{lcc}
\hline \hline & cc-pVDZ & cc-pVTZ \\
\hline Triad & & \\
N 1 & 129.9 & 134.6 \\
N 2 & 166.9 & 171.8 \\
N 3 & 217.1 & 224.4 \\
N 4 & 457.4 & 474.7 \\
N 5 & 164.3 & 170.5 \\
N 6 & 91.4 & 95.6 \\
O 1 & 180.8 & 187.5 \\
O 2 & 400.3 & 406.3 \\
O 3 & 404.3 & 406.8 \\
O 4 & 364.2 & 366.9 \\
O 5 & 444.4 & 446.0 \\
\hline
\end{tabular}

Building eq. (26)

CPU (elapsed) time / $\min 30.1(30.7) \quad 99.3(130.2)$

CPHF iteration step

CPU (elapsed) time / min 26.6 (27.2) $\quad 71.0$ (141.6)

DF-HF iteration step

CPU (elapsed) time / min 28.1 (28.4) 97.6 (98.9)

a) $\mathrm{NH}_{3}$ optimized at MP2/cc-pVTZ level, absolute chemical shifts 278.3 (cc-pVDZ), 269.2 (cc-pVTZ).

b) $\mathrm{H}_{2} \mathrm{O}$ optimized at MP2/cc-pVTZ level, absolute chemical shifts 347.2 (cc-pVDZ), 334.9 (cc-pVTZ). 